\title{
Des enzymes peuvent servir de protéines de structure dans le cristallin
}

Les constituants principaux du cristallin sont des protéines solubles, les cristallines. Elles représentent 20 à $50 \%$ du poids sec de l'organe et sont d'une extraordinaire stabilité physique et métabolique, puisqu'elles sont soustraites au renouvellement général des protéines de l'organisme. Chez les mammifères, ce groupe est formé des cristallines, $\alpha, \beta$ et $\gamma$. On trouve chez les oiseaux et les reptiles une $\delta$-cristalline, et nombre de représentants de ces classes contiennent de l' $\varepsilon$-cristalline. D'autres formes, dites $\rho$ et $\tau$, existent en outre dans certaines espèces. Chacune de ces cristallines est codée par un gène particulier.

La multiplication des séquences géniques et protéiques connues a permis souvent de découvrir des homologies, et parfois des identités, inattendues, et aussi de raccorder des protéines à un ancêtre commun, définissant ainsi des familles et des superfamilles de protéines. Dans le cas des cristallines, on a eu la surprise de découvrir que certaines d'entre elles ressemblent à des enzymes. Mais s'agit-il d'identité ou de « descendance » ? A cette question il est souvent encore difficile de répondre lorsque l'enzyme et la cristalline n'ont pas été étudiées dans la même espèce : par exemple, on connaît les séquences d'une cristalline de grenouille et de l'enzyme provenant de poumon humain ou de levure; la réponse définitive est une question de temps.

Il est toutefois un exemple où l'identité a pu être démontrée sans équivoque : c'est celui de l' $\varepsilon$-cristalline. Elle est présente dans le cristallin de certains oiseaux et reptiles à des taux qui atteignent 10 à $25 \%$ du total, notamment chez le canard et le crocodile. L'analyse de la protéine avait fait conclure à une équipe néerlandaise [1] que l' $\varepsilon$-cristalline et la lacticodeshydrogénase type cœur (LDH B) se ressemblaient, et que cette cristalline possédait une activité LDH. Le clonage de l'ADN complémentaire de l' $\varepsilon$-cristalline par la même équipe [2] fut réalisé en criblant une banque d'expression avec un sérum anticristalline $\varepsilon$. La séquence nucléotidique fut trouvée identique à celle de la LDH B ; dans la séquence des acides aminés, les seules différences - deux asparagines remplacées dans la cristalline par des aspartates proviennent de désamidations posttraductionnelles de deux restes d'asparagines, phénomène bien connu lors du vieillissement des protéines du cristallin. Ajoutons qu'il n'existe qu'un seul gène pour le couple $\mathrm{LDH}$ B- $\varepsilon$-cristalline, comme on peut le vérifier par la méthode de Southern. Qu'une enzyme comme la $\mathrm{LDH}$ puisse former une protéine de structure en proportions abondantes est donc démontré. Bien des questions restent cependant obscures. Pourquoi la LDH ? On sait que l'isozyme B est plus stable que la forme A, et aussi que les LDH d'oiseaux sont plus stables (notamment à la chaleur) que celles des mammifères. Reste le caractère capricieux de la présence d'E-cristalline selon les espèces. Les mêmes auteurs [1] ont recherché dans quelles espèces l' $\varepsilon$ cristalline était exprimée dans le cristallin. Parmi les oiseaux, on la trouve chez la mouette, le pélican, la cicogne, le flamant, le canard, mais non chez le poulet, le moineau, le pigeon, la chouette.

Aucune autre identité n'a été démontrée entre une enzyme et une cristalline. Il existe cependant d'autres exemples potentiels. Le plus probant a été fourni par une équipe japonaise. Elle avait décrit en 1981 dans le poumon de rat une enzyme qu'elle a appelée prostaglandine $F$ synthétase. Le clonage récent de son $\mathrm{ADNc}[3]$ à partir de poumon de bœuf montre une homologie très importante avec une cristalline de la grenouille (Rana temporaria), confondue initialement avec la forme $\varepsilon$, mais appelée actuellement $\rho$-cristalline. Les deux protéines ont en effet $77 \%$ d'identité, et compte tenu de l'écart entre la grenouille et les mammifères, il est vraisemblable qu'on puisse aboutir à la conclusion que la PGF synthétase et la $\rho$-cristalline sont identiques. Ces protéines présentent en outre des homologies très nettes avec une aldéhyde réductase humaine et font sans doute partie de la même famille.

Le concept de l'identité, ou des relations familiales, entre enzymes et cristallines a été généralisé par Wiston et Piatigorshy $[4,5]$. La $\delta$-cristalline est la protéine majeure du cristallin embryonnaire de tous les oiseaux et reptiles. La cristalline de poulet présente des homologies importantes (environ $60 \%$ ) avec une enzyme du cycle de l'urée, l'argininosuccinate lyase, aussi bien de la levure que de l'homme. La $\tau$-cristalline, présente chez certains oiseaux, reptiles, poissons et lamproies, ressemble à l'énolase humaine et de levure, bien que l'activité d'énolase de cette cristalline soit faible. Enfin, les protéines les plus abondantes du cristallin de calmar sont très voisines, et même peut-être identiques, à des formes de glutathion-S-transférases [6].

Comment interpréter cette variété de résultats qui montrent une parenté indiscutable entre des protéines de 
structure et des enzymes, dont le rôle en tant que telles est parfaitement superflu dans le cristallin? Cet organe est d'apparition relativement récente dans l'évolution. On peut supposer que certaines enzymes aient été « recrutées » [4] en raison, par exemple, d'une structure particulièrement stable et du fait qu'à forte concentration elles n'entravaient pas le passage de la lumière. Selon les classes de vertébrés et même selon les espèces, le choix a pu être différent. Par la suite, dans certains cas, le gène est resté unique et bifonctionnel, comme pour la LDH B et l' $\varepsilon$-cristalline ; dans d'autres, il s'est sans doute dupliqué avec évolution divergente des deux gènes après leur séparation. Les enzymes ne paraissent, en revanche, pas être à l'origine des trois cristallines $\alpha, \beta$ et $\gamma$. Elles n'en ont pas moins des apparentements : l' $\alpha$ cristalline appartient à la même superfamille que les petites protéines du choc thermique et qu'un antigène de l'œuf de Schistosoma mansoni, une douve; quant à $\beta$ et $\gamma$, elles font partie d'une superfamille qui pourrait inclure la protéine $S$ liant le calcium d'une bactérie, Myxococcus xanthus [5].

Protéines très spécialisées, les cristallines, réservent sans doute encore bien des surprises.

J.-C. D.

1. Wistow.GT, Mulders JWM, de JONG WW. The enzyme lactate dehydrogenase as a structural protein in avian and crocodilian lenses. Nature $1987 ; 326: 622-4$

2. Hendriks W, Mulders JWM, Bibby MA, et al. Duck lens $\varepsilon$-crystallin and lactate dehydrogenase B4 are identical: a single-copy gene product with two distinct functions. Proc Natl Acad Sci USA $1988 ; 85$ : 7114-8.

3. Watanabe K, Fujii Y, Nakayama K, et al. Structural similarity of bovine lung prostaglandin $F$ synthase to lens $\varepsilon$-crystallin of the european common frog. Proc Natl Acad Sci USA $1988 ; 85: 11-5$.

4. Wistow G, Piatigorsky J. Recruitment of enzymes as lens structural proteins. Science 1987; 236 : 1554-6.

5. Wistow G, Piatigorsky J. Lens crystallins : the evolution and expression of proteins for a highly specialized tissue. Annu Rev Biochem $1988 ; 57: 479-504$.

6. Tomarev SI, Zinovieva RD. Sq id major lens polyveptides are homologou. to glutathion-S-transferases subunits. Nature 1988;
Récepteurs aux benzodiazépines et foyers épileptiques. La localisation précise d'un foyer épileptique est importante, surtout lorsqu'on en envisage l'ablation. Elle est souvent difficile, même par encéphalographie intracérébrale stéréotaxique, méthode invasive qui n'est pas dépourvue d'inconvénients. On s'est donc tourné vers un principe différent : le récepteur des benzodiazépines (BZ) est un marqueur du complexe du récepteur du GABA (acide $\gamma$-aminobutyrique) (voir $\mathrm{m} / \mathrm{s}$ $n^{\circ} 1$, vol. $3, p .45$ ), qui est probablement impliqué dans l'épilepsie. Une équipe américaine [1] a étudié dix malades atteints d'épilepsie partielle, après avoir localisé la lésion le mieux possible par électro-encéphalographie, ils ont utilisé la tomographie à émission de positons. Le réactif employé était l'antagoniste des récepteurs des BZ appelé Ro-/15 1788, marqué par l'émetteur de positons

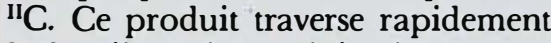
la barrière hémo-méningée et ne se lie qu'aux neurones et non au tissu glial. Chez tous les malades, la concentration du marqueur dans le foyer présumé était inférieure de 15 à $35 \%$ à celle de la région de référence, alors que la variation ne dépassait pas $4 \%$ chez les témoins. Ces résultats renforcent l'hypothèse que la transmission inhibitrice est perturbée dans un foyer épileptique. Le récepteur des $\mathrm{BZ}$ devrait donc pouvoir être utilisé pour la détection des foyers épileptiques par cette méthode, qui semble plus précise que celle au désoxyglucose [2]. D'autres auteurs, il est vrai, travaillant in vitro, n'ont trouvé qu'une faible diminution de la liaison des BZ [3]. Ces travaux sont toutefois délicats à interpréter du fait de l'insuffisance de tissu normal de référence. Ces objections ne devraient pas enlever leur valeur aux recherches pratiquées in vivo, et on peut espérer que les progrès des méthodes d'émission de positons parviendront à localiser exactement les foyers dans les cas graves où une exérèse peut être indiquée.

[1. Savic I, et al. Lancet 1988; ii : 863-6.]
[2. Engel J, et al. Neurology 1983 ; 33 : 400-13.]

[3. Sherwin A, et al. Neurology 1986 ; $36: 1380-2$.]

Les glucocorticoïdes stimulent directement l'expression du gène à l'ANF. Cela est dû surtout à l'augmentation de la transcription dans les cellules exprimant le gène, et non au recrutement de cellules quiescentes (comme l'ont montré les expériences d'hybridation in situ).

[Gardner DG, et al. J Clin Invest $1988 ; 82$ : 1275-81.]

Trois facteurs en un. Le facteur inhibiteur des leucémies myéloïdes (LIF, myeloïd leukemia inhibitory factor), l'activité inhibitrice de la différenciation des cellules souches embryonnaires totipotentes ES (DIA, differenciation inhibitory activity) et l'interleukine active sur les cellules DA (HILDA, human interleukin for $D A$ cells) correspondent au produit d'un seul et même gène dont l'ADN complémentaire a été cloné [1-3].

Le DIA, purifié à partir d'un milieu de culture conditionné par des cellules hépatiques de rat Buffalo, permet de cultiver des cellules ES sans sous-couche nourricière tout en leur conservant leur totipotence. Le HILDA permet de maintenir en culture des cellules leucémiques particulières (cellules DA). Le LIF induit in vitro la différenciation de cellules myéloïdes leucémiques en monocytes. Ainsi, selon la cellule cible, cette molécule. (codée par un messager de 3,8 kilobases ayant une séquence traductible en une protéine de 202 acides aminés) se comportet-elle comme un inhibiteur... ou un inducteur de différenciation.

[1. Williams LR, et al. Nature 1988 ; 336 : 684-7.]

[2. Smith AG, et al. Nature 1988 ; 336 : 688-90.]

[3. Moreau JF, et al. Nature 1988; 336 : 690-2.] 